\title{
Cygnus X-1: shedding light on the spectral variability of the hard state of black holes
}

\author{
Victoria Grinberg $^{* 1}$, Diana M. Marcu ${ }^{2,3,4}$, Katja Pottschmidt ${ }^{2,3}$, Moritz Böck ${ }^{1}$, Jörn \\ Wilms $^{1}$, Marion Cadolle Bel ${ }^{5}$, Anne M. Lohfink ${ }^{2,3,6}$, Felix Fürst ${ }^{1}$, Manfred Hanke, ${ }^{1}$, \\ Michael A. Nowak ${ }^{7}$, Sera B. Markoff ${ }^{8}$, Alex Markowitz ${ }^{9}$, John A. Tomsick ${ }^{10}$, Jérôme \\ Rodriguez $^{11}$, Guy G. Pooley ${ }^{12}$, Isabel Caballero ${ }^{11}$
}

*E-mail: Victoria.Grinberg@sternwarte.uni-erlangen. de

${ }^{1}$ Remeis-Observatory/ECAP/FAU, Bamberg, Germany

${ }^{2}$ CRESST/NASA-GSFC, Greenbelt, MD, USA

${ }^{3} U M B C$, Baltimore, MD, USA

${ }^{4}$ GMU, Fairfax, VA, USA

${ }^{5}$ ESAC, Madrid, Spain

${ }^{6}$ UMCP, College Park, MD, USA

${ }^{7}$ MIT Kavli Institute, Cambridge, MA, US

${ }^{8}$ UVA, Amsterdam, NL

${ }^{9}$ UCSD, La Jolla, CA, USA

${ }^{10}$ SSL, UC Berkeley, Berkeley, CA, USA

${ }^{11}$ DSM/DAPNIA/SAp, CEA Saclay, France

${ }^{12}$ University of Cambridge, Cambridge, UK

We present an analysis of extensive recent monitoring observations of the black hole X-ray binary Cygnus X-1 obtained as part of the 2007 to 2010 Cygnus Region Key Programme observations of the INTEGRAL mission. Cyg X-1 is one of only three persistent black hole binaries in our galaxy that spend most of their time in the hard spectral state. We concentrate on constraining the parameter range of the hard spectrum, a measurement that is typically difficult to obtain with high accuracy for transient sources, but which is important to know in order to understand the physics of the hot plasma of the jet base and/or the corona. While the hard X-ray spectrum of Cyg X-1 is one of the best studied examples of its kind, e.g., through our years long monitoring campaign with RXTE, the INTEGRAL monitoring allows us to study the spectral evolution from about half an hour over a few days to a few weeks, timescales that have been in part only sparsely sampled so far. After spending $\sim 3$ years in the hardest regime of its parameter space, the source displayed a softening and flaring episode in mid 2009 and entered a soft state in early 2010 June. We compare X-ray broad band spectra (RXTE, INTEGRAL) of these two emission states. Furthermore, we use INTEGRAL/IBIS to extend the timing analysis with a resolution of up to $0.1 \mathrm{~s}$ to energies above $20 \mathrm{keV}$.

8th INTEGRAL Workshop "The Restless Gamma-ray Universe"

September 27-30 2010

Dublin Castle, Dublin, Ireland 


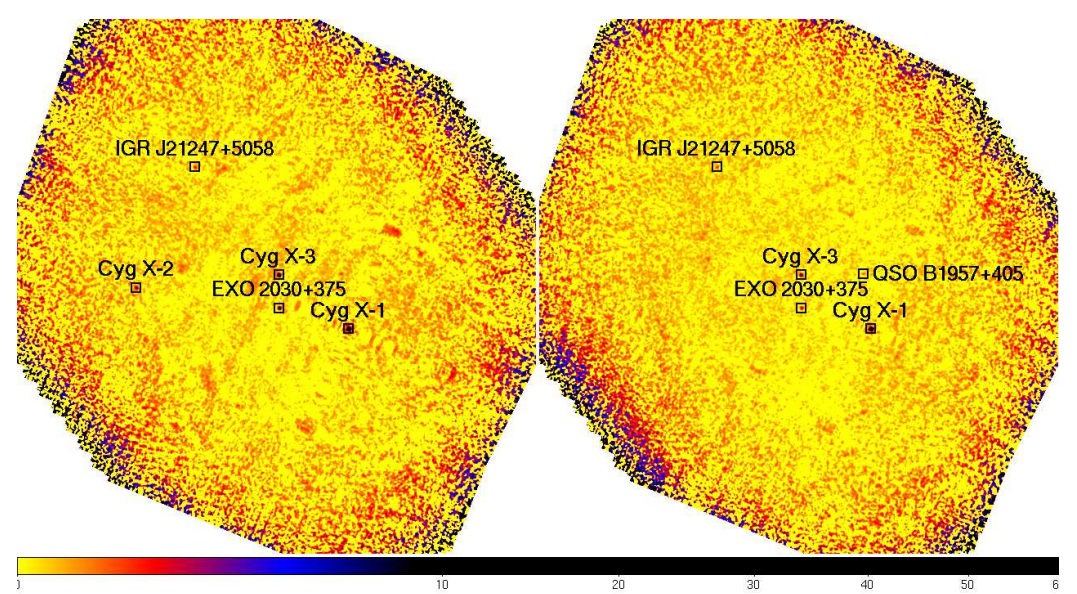

Figure 1: Intensity mosaic for the Cyg X-1 field in the $20-40 \mathrm{keV}$ (left) and $40-80 \mathrm{keV}$ (right) energy bands for the KP revolutions 803-806. Named boxes indicate sources detected with $\sigma_{\mathrm{det}} \geq 6$.

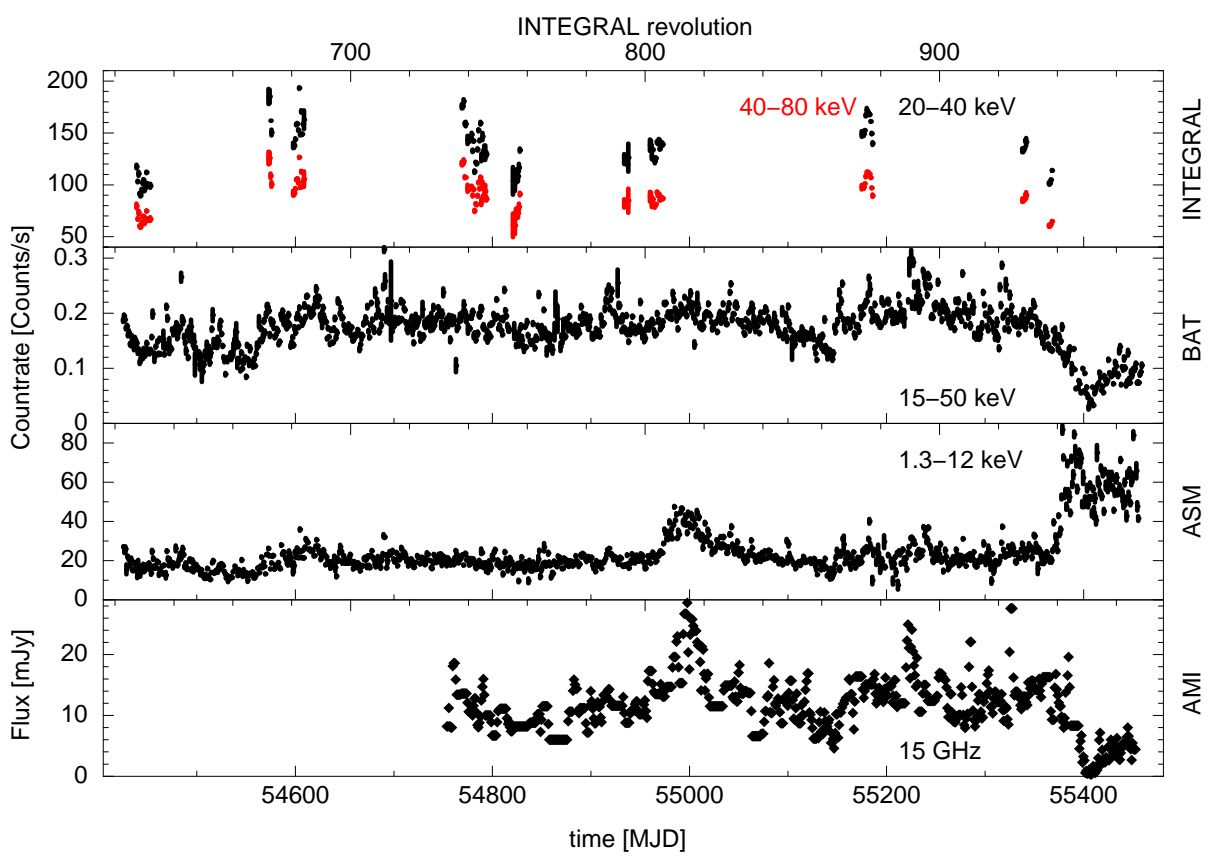

Figure 2: INTEGRAL, Swift/BAT, RXTE/ASM and AMI lightcurves of Cyg X-1 during the KP.

\section{Introduction: The Cyg X-1 Key Programme}

Cyg X-1 is a persistent, bright high mass X-ray binary, which spends most of the time in the hard/low state, but shows excursions into the soft/high state. The source has been observed as part of the INTEGRAL Key Programme (KP) since 2007. An example intensity mosaic of the Cyg X-1 field from the KP is shown in Fig. 1.

Within the ongoing KP fall a long, extraordinarily hard state (2006 to spring 2010, see [5]), a short radio flaring episode in spring 2009 (about MJD 54950-55050, KP revolutions 803-806 cover the beginning) and, since summer 2010, a soft state (KP revolution 938 covers the transition 

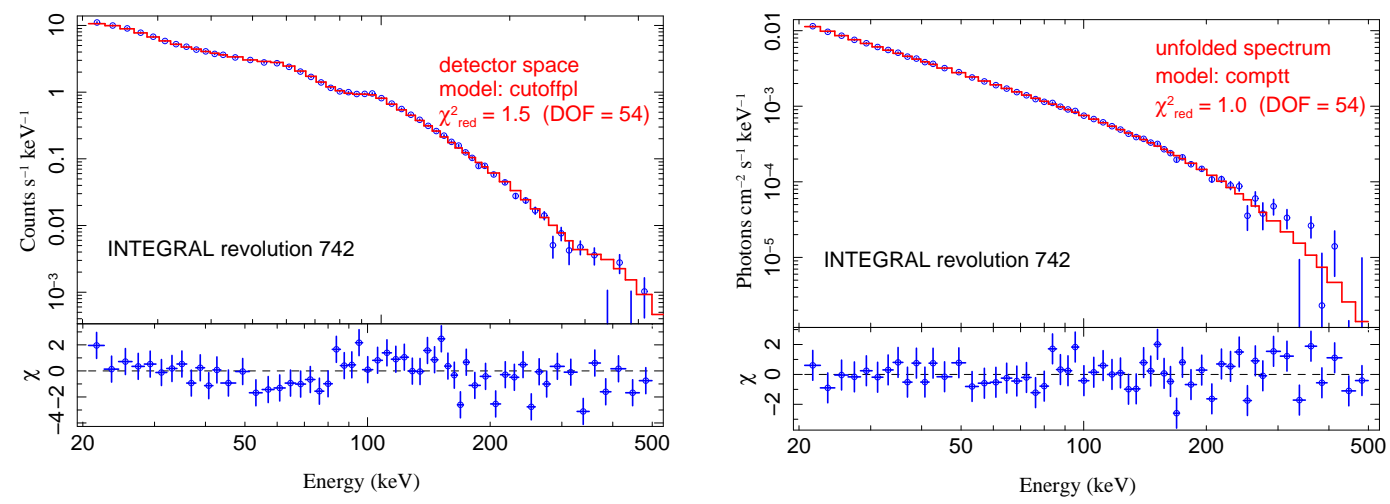

Figure 3: cut offpl (left) and comptt (right) fits to Cyg X-1 spectra from KP revolution 742.

into the soft state). Lightcurves of Cyg X-1 taken with different instruments since MJD 54500 are presented in Fig. 2. While the radio flaring episode of spring 2009 was observed in the soft X-rays with RXTE/ASM, there is no indication of extraordinary activity in the Swift/BAT energy range. The soft state of summer 2010 is clearly visible both in the soft (increased ASM count rate) and hard X-rays (decreased BAT count rate).

\section{Spectral modelling and evolution of the spectal parameters}

We extracted spectra for all the Science Windows (ScWs) in the Cyg X-1 KP using OSA 9. To achieve a better signal to noise ratio all spectra from a given INTEGRAL revolution ( $\sim 3$ days) were then added. Following the recommendation of the IBIS cookbook ${ }^{1}$, a systematic uncertainty of $2 \%$ was used when fitting.

Both the empirical cutoff power law and simple thermal Comptonization models (cutoffpl and comptt, respectively) allow for good fits to the ISGRI data in the $20-500 \mathrm{keV}$ range, as can be seen from the example presented in Fig. 3. In RXTE observations the temperature of the seed photons for the comptt model has been previously constrained to a range around roughly $1 \mathrm{keV}$ [8]. This parameter can not be constrained in the ISGRI data and has been frozen to a value of $1 \mathrm{keV}$. Fitting this parameter neither significantly improves the fit statistics nor causes changes in other model parameters. Both models usually result in fits of comparable quality (see Fig. 4 for $\chi_{\text {red }}^{2}$ values). Especially, we do not see the instrumental line at $\sim 60 \mathrm{keV}$, which has been reported for previous versions of the OSA software [6].

Figure 4 shows the evolution of the spectral parameters on the timescale of single revolutions. Although there is a clear rising trend in the values of the reduced $\chi^{2}$, no systematic contribution to the residuals persisting over several revolutions is apparent. It can be assumed that this trend can be attributed to time dependencies which are not taken into account by the current response.

The best fit values for the revolutions 803-806 (MJD 54962-54973, 10.5.2009.-22.5.2009) do not show any deviations from the values observed for other revolutions in the prolonged hard state. This result agrees with the fact that the radio flare of spring 2009 is not seen in the BAT energy range, i.e. the flaring episode does not show typical transitional behaviour.

\footnotetext{
${ }^{1}$ See http://isdcul3.unige.ch/Soft/download/osa/osa_doc/osa_doc-9.0/osa_um_ ibis-9.2/index.html
} 


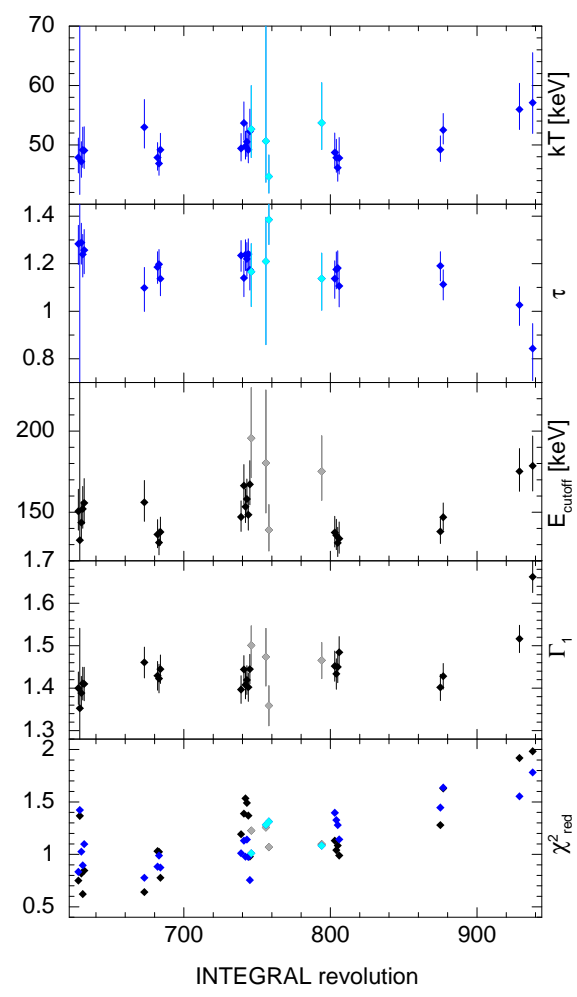

Figure 4: Fit parameters of the best fits with cutoffpl (black) and comptt (blue) models for the observations of Cyg X-1 during the time of the Cyg X-1 KP. Grey and cyan points signify revolutions with less than $25 \mathrm{ScWs}$ of Cyg X-1 observations and therefore less well constrained spectra.

Table 1: Best fit parameters for simultaneous RXTE and INTEGRAL/ISGRI observations of Cyg X-1 during the 2009 soft episode (RXTE Obs-ID 94121-01-00-00 and INTEGRAL revolution 803).

\begin{tabular}{l|c|c|c} 
instrument & $\Gamma_{1}$ & $\Gamma_{2}$ & $E_{\text {fold }}[\mathrm{keV}]$ \\
\hline PCA \& HEXTE & $1.78 \pm 0.10$ & $1.48 \pm 0.02$ & $197 \pm 20$ \\
PCA, HEXTE \& ISGRI & $1.77 \pm 0.10$ & $1.48 \pm 0.02$ & $143 \pm 4$
\end{tabular}

The fit parameters clearly differ only for the two observations in spring 2010 (revolutions 929 and 938), which were both conducted during the beginning of the transition to the soft state. Note that both revolutions do not fall within the soft state itself. In terms of the cut of fpl parameters the slope steepens and the cutoff energy $E_{\text {cut }}$ increases. In the Comptonization description the optical depth $\tau$ decreases and the plasma temperature $k T$ increases. These trends are consistent with previous INTEGRAL results [1] as well as the spectral evolution as seen by RXTE [8].

During the spring 2009 radio flare episode simultaneous RXTE and INTEGRAL/ISGRI data are available. In Fig. 5 and Tab. 11 we present a fit of these data using the RXTE Obs-ID 9412101-00-00 and the INTEGRAL revolution 803 as an example. We employ a broken power law model modified by an exponential cutoff and a Gaussian at $6.4 \mathrm{keV}$ to describe the $\mathrm{Fe} \mathrm{K} \alpha$ line. Additionally the interstellar absorption is taken into account.

The best fit parameters for the slope for the RXTE only and the RXTE and INTEGRAL spectra fitted together agree well within the respective uncertainities. The value of the power law slope at energies above $10 \mathrm{keV}$ also agrees with the INTEGRAL only results from above. The value of the cutoff energy changes strongly between RXTE only and combined RXTE and INTEGRAL data. Given the additional data points at high energies a change in this parameter is not unexpected. 

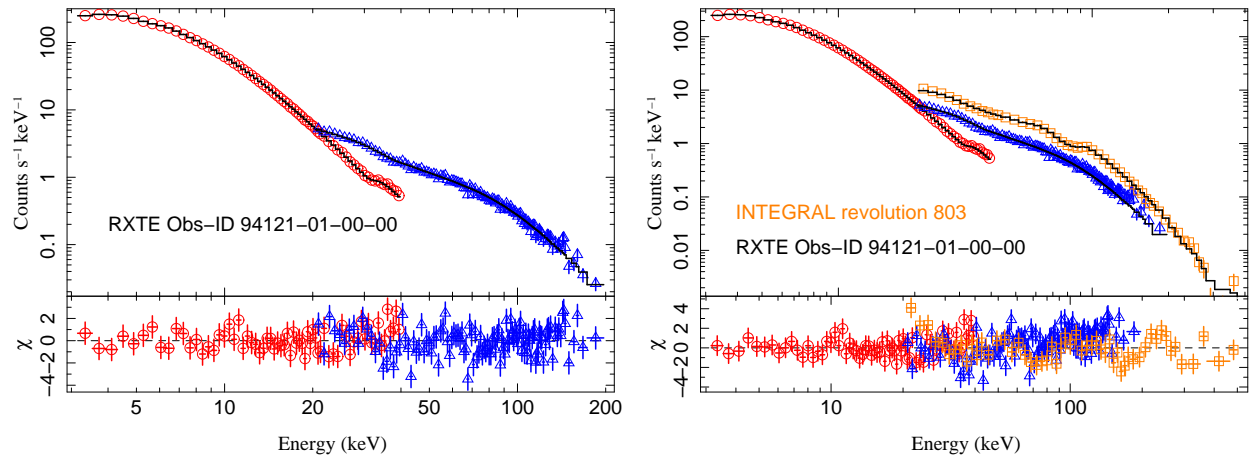

Figure 5: Best fit broken power law models for simultaneous RXTE (PCA: red circles and HEXTE: blue triangles) and INTEGRAL/ISGRI (brown boxes) observations of Cyg X-1 during the spring 2009 soft episode.

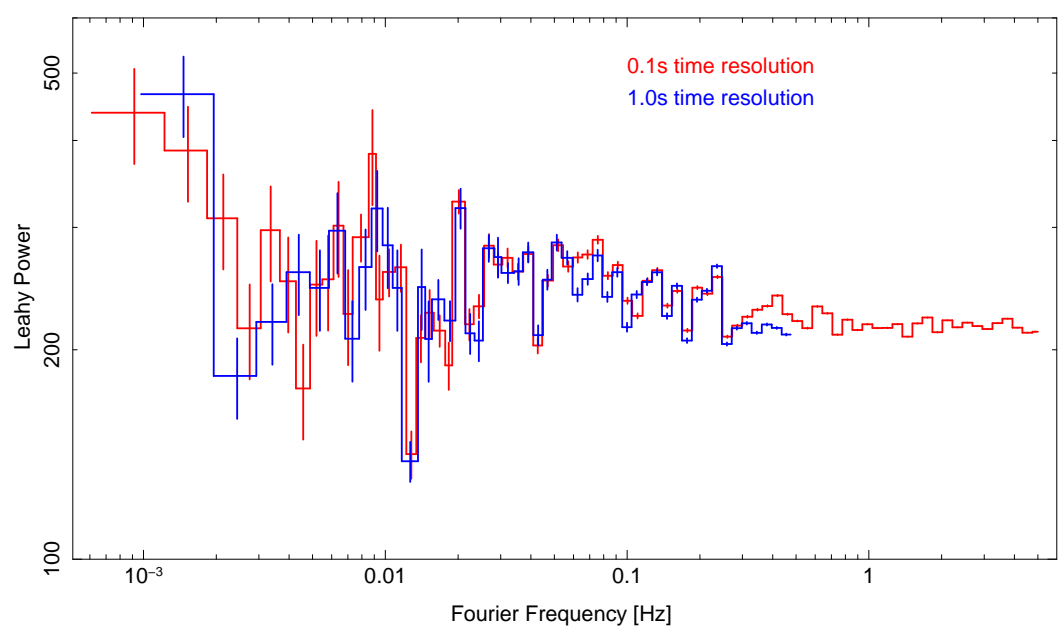

Figure 6: Leahy-normalized [卂] PSDs of Cyg X-1 calculated from lightcurves created with the ii_light algorithm.

The value for the combined fit of $\sim 140 \mathrm{keV}$ agrees well with the INTEGRAL only value from above. This strengthens the notion that no effect of the radio flare is seen at energies of $20-500 \mathrm{keV}$.

The latest Cyg X-1 KP observations available at the time of the analysis presented here cover only the very beginning of the transition to the soft state. A first analysis of the RXTE observations of the soft state have been presented by [3]. Note that only the use of both timing and spectral properties allows for an exact state characterisation [3, 元].

\section{X-ray Timing Analysis with INTEGRAL}

The use of the ii_light algorithm for lightcurve extraction allows us to achieve a time resolution of up to $0.1 \mathrm{~s}$ for sources as bright as Cyg X-1 ( $\sim 100 \mathrm{cps}$ with ISGRI in the $20-40 \mathrm{keV}$ band). Here we present PSDs calculated in the $20-40 \mathrm{keV}$ energy band on ii_light lightcurves with $1 \mathrm{~s}$ and $0.1 \mathrm{~s}$ time resolution for the revolution 742. As Fig. 6 shows, the PSDs agree well with each other. The PSDs are shown in Leahy normalisation [П], where the Poisson noise level should be equal to $2 \mathrm{rms}^{2} / \mathrm{Hz}$ independent of source properties. This is clearly not the case, instead the PSDs flatten out at values of $\sim 200 \mathrm{rms}^{2} / \mathrm{Hz}$. In their analysis of Vela X-1 Fürst et al. [2] re- 
ported a Poisson noise contribution of $100 \mathrm{rms}^{2} / \mathrm{Hz}$ when using OSA 7 and ii_light generated lightcurves with $6 \mathrm{~s}$ time resolution. They have also shown that in spite of the anomalous noise the characteristic frequencies are well reproduced. The higher anomalous noise contribution in the presented PSDs might be either due to a different software version or the different source field or a combination of both. The shape of the INTEGRAL PSDs can therefore be used for the source variability analysis, allowing us to extend the timing analysis to higher energies, but the behaviour of the anomalous noise has to be investigated further.

\section{Summary}

We have analysed the spectral evolution of Cyg X-1 as observed with INTEGRAL/IBIS during the INTEGRAL Cyg X-1 KP. The source spectrum is stable in the IBIS energy range during the prolonged hard state as well as the radio flare and stepens as the source enters the soft state. During the radio flare, RXTE data agree with the spectral parameters derived from INTEGRAL data only The timing analysis suffers from problems with anomalous noise, but using the shape of the PSDs should allow to extend the analysis of the source variability into the IBIS range.

\section{Acknowledgments}

This research was funded by the Bundesministerium für Wirtschaft und Technologie under Deutsches Zentrum für Luft- und Raumfahrt grant number 50 OR 1007 and partially funded by the European Commission under contract ITN 215212 "Black Hole Universe".

\section{References}

[1] M. Cadolle Bel, P. Sizun, A. Goldwurm, J. Rodriguez, P. Laurent, A. A. Zdziarski, L. Foschini, P. Goldoni, C. Gouiffès, J. Malzac, E. Jourdain, and J.-P. Roques, The broad-band spectrum of Cygnus X-1 measured by INTEGRAL, A\&A 446, 591-602 (2006).

[2] F. Fürst, I. Kreykenbohm, K. Pottschmidt, J. Wilms, M. Hanke, R. E. Rothschild, P. Kretschmar, N. S. Schulz, D. P. Huenemoerder, D. Klochkov, and R. Staubert, $X$-ray variation statistics and wind clumping in Vela $X-1, \mathrm{~A} \& \mathrm{~A}$ 519, A37 (2010).

[3] V. Grinberg, M. Boeck, K. Pottschmidt, M. A. Nowak, P. Uttley, J. A. Tomsick, A. Bodaghee, S. Markoff, G. Pooley, I. Kreykenbohm, and J. Wilms, RXTE Monitoring of Cyg X-1 in its current transitional state, The Astronomer's Telegram 2751 (2010).

[4] D. A. Leahy, W. Darbro, R. F. Elsner, M. C. Weisskopf, S. Kahn, P. G. Sutherland, and J. E. Grindlay, On searches for pulsed emission with application to four globular cluster X-ray sources - NGC 1851, 6441, 6624, and 6712, ApJ 266, 160-170 (1983).
[5] M. A. Nowak, M. Hanke, S. N. Trowbridge, S. B. Markoff, J. Wilms, P. Coppi, D. Maitra, J. E. Davis, F. Tramper, Corona, jet, and relativistic line models for Suzaku/RXTE/Chandra-HETG observations of the Cygnus X-1 hard state, ApJ, submitted (2010).

[6] K. Pottschmidt, D. M. Marcu, V. Grinberg, J. Wilms, M. Cadolle Bel, A. Lohfink, F. Fuerst, M. Hanke, M. A. Nowak, S. Markoff, J. A. Tomsick, J. Rodriguez, and G. G. Pooley, Cygnus X-1: Shedding Light On The Spectral Variability Of The Hard State Of Black Holes in proceedings of American Astronomical Society, HEAD meeting \#11, , Bulletin of the American Astronomical Society, vol. 41, February 2010, p. 671.

[7] K. Pottschmidt, J. Wilms, M. A. Nowak, G. G. Pooley, T. Gleissner, W. A. Heindl, D. M. Smith, R. Remillard, and R. Staubert, Long term variability of Cygnus $X$-1. I. X-ray spectral-temporal correlations in the hard state, A\&A 407, 1039-1058 (2003).

[8] J. Wilms, M. A. Nowak, K. Pottschmidt, G. G. Pooley, and S. Fritz, Long term variability of Cygnus X-1. IV. Spectral evolution 1999-2004, A\&A 447, 245-261 (2006). 